\title{
Reviewing the psychotic spectrum approach: a continuum or separate diseases? Konstantinos N Fountoulakis
}

\author{
Address: 3rd Department of Psychiatry, Aristotle University of Thessaloniki, Greece \\ from International Society on Brain and Behaviour: 3rd International Congress on Brain and Behaviour \\ Thessaloniki, Greece. 28 November - 2 December 2007 \\ Published: 17 April 2008 \\ Annals of General Psychiatry 2008, 7(Suppl I):S64 doi:I0.I I86/I744-859X-7-SI-S64
}

This abstract is available from: http://www.annals-general-psychiatry.com/content/7/SI/S64

(C) 2008 Fountoulakis; licensee BioMed Central Ltd.

Since Kraepelin distinguished dementia praecox from manic-depressive illness, the generally accepted opinion among psychiatrists is that there are two distinct major psychotic illnesses, characterized by both psychodynamic and biological differences, responding to different treatments and with a very different prognosis. However the 'unitary psychosis' theory has never been completely abandoned. The recent advances in the understanding of these two disorders revived the century-old debate. Clinical data seem to favour the presence of a continuum with schizoaffective syndromes in the middle rather than the existence of two distinct diseases. The recognition of residual symptoms and a neurocognitive deficit in bipolar patients brings them even closer. The treatment has never before been more similar and both disorders demand the use of combination therapy of almost all available psychotropic agents in order to achieve the best possible outcome. However important differences also emerged. Patients with schizophrenia tend to manifest neurocognitive deficits and decline in functioning early since adolescence while bipolar patients manifest a higher than average functioning during the same age periods. The neurocognitive deficit is rather different although the clinical picture shares common features, key clinical elements differ. Genetic and other biological data are mixed but rather point to a syndromal rather than a trait and disease specific dysfunction. Conclusively, data suggest the presence of two distinct poles but it is uncertain what the interpretation for the in-between cases and states should be. 